\title{
3D imaging in orthodontics and dentofacial orthopaedics
}

\author{
Imran Nizam Pathan
}

Assistant Professor in the department of dentistry in Government Medical College and hospital, Jalgaon-425001, Maharashtra, INDIA. Email: imran352@gmail.com

Abstract Background: Orthodontist job is to fit together the pieces of the craniofacial complex puzzle. Orthodontic treatment affects the craniofacial relationship in 3 planes of space. Yet strangely enough, the critical diagnostic records currently used are 2dimensional. In 3D imaging a set of anatomical data is collected using diagnostic imaging equipment, processed by computer and then displayed on a 2D monitor to give the illusion of depth. The goal of 3D imaging is to create a life like digital record of Human dentofacial morphology.

Keywords: Cone beam, tomography, imaging, orthodontics, three-dimensional

*Address for Correspondence:

Dr.Imran Nizam Pathan, Assistant Professor in the department of dentistry in Government Medical College and hospital, Jalgaon-425001,

Maharashtra, INDIA.

Email: imran352@gmail.com

Received Date: 10/11/2019 Revised Date: 24/12/2019 Accepted Date: 15/01/2020

DOI: https://doi.org/10.26611/10131331

\begin{tabular}{|l|l|}
\hline \multicolumn{2}{|c|}{ Access this article online } \\
\hline Quick Response Code: & \multirow{2}{*}{ Website: } \\
& www.medpulse.in \\
\cline { 2 - 2 } & \\
\hline
\end{tabular}

\section{INTRODUCTION}

Three-dimensional (3D) imaging has evolved greatly in the last two decades and has found its applications in orthodontics. ${ }^{1}$ The correction of dentofacial deformities requires teeth and jaws to be manipulated in 3 dimensions to achieve the best results within the constraints of aesthetics, stability, and function. ${ }^{2}$ Our interest is in the totality of craniofacial anatomy and in the interactions among the parts. ${ }^{3}$ It also allows the clinician to monitor and document the treatment progress and outcome. ${ }^{4}{ }^{14}$ The use of lateral cephalometric radiographs forms an important diagnostic tool in orthodontics treatment as well as orthognathic surgery. However, their 2 dimensional nature presents an inherent limitation to the clinician, as the human body is 3 dimensional. ${ }^{19}$ In addition, a significant amount of radiographic projection error further limits their accuracy. Three-dimensional imaging of the human body via computed tomography has been available to the field of medicine for the last 30 years. However, the significant amount of radiation exposure associated with this technology, precluded its widespread use in dentistry ${ }^{20}$. With the development of Cone Beam Computed Tomography, there has been a drastic reduction in radiation exposure to the patient, which allows its use for safely obtaining 3 dimensional images of the craniofacial structures. ${ }^{15}$ This should allow the clinician to visualize the hard and soft tissues of the craniofacial region from multiple perspectives, which could have far-reaching implications for treatment planning in orthodontics and orthognathic surgery. ${ }^{7}$

\section{Classifications}

i] Udupa and Herman classified 3D imaging approaches into three categories: ${ }^{1}$

1. Slice imaging- e.g. a set of $\mathrm{CT}$ axial data to produce reconstructed 2D images.

2. Projective imaging- e.g. surface laser scanning to produce what is considered a 2.5-D mode of visualization;

3. Volume imaging- e.g. holography or 'varifocalmirrors'techniques.

ii] 3D imaging in Orthodontics can be broadly classified as. ${ }^{12}$ :
1) Facial imaging
2) Dental imaging
3) Skeletal imaging 
1. Facial imaging:-

Stereophotogrammetry

What is Stereophotogrammetry?

It refers to the special case where two cameras, configured as a stereo pair, are used to recover 3D distances of features on the surface of the face by means of triangulation. ${ }^{1}$

\section{Armamentarium}

It consists of two synchronized semi-metric cameras mounted on a frame with a distance of $50 \mathrm{~cm}$ between them and positioned convergently with an angle of $15^{\circ}$.

\section{Orthodontic application:-}

The incorporation of recent technology in computer science in the field of stereophotogrammetry has given the ability to process complex algorithms in order to convert simple photographs to three-dimensional measurements of facial changes. ${ }^{16}$

It gives the three-dimensional coordinates of any chosen facial landmark, so linear and angular measurements could be calculated to detect any changes in facial morphology. ${ }^{26}$ It also gives the clinician a life-like 3D model of the patient's head that can be rotated, enlarged, and measured in three dimensions as required for diagnosis, treatment planning and surgical outcome analysis. ${ }^{13,24}$

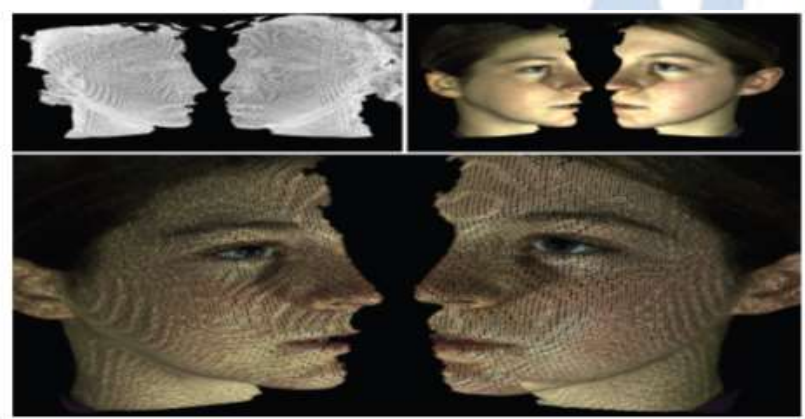

Figure 1. The whole merged 3D face is a composite of two halves, each half representing the image acquisition from each pod. Top left: wireframe range models without any surface texture. Top right: facia texture maps of both sides. Bottom: 3D rendered polygonal meshes with textures enforced. ${ }^{1}$

Applications of 3D imaging of the face:-

Assessment of facial deformity and the outcome of surgical and/or orthodontic correction: For the subjective assessment of deformities, 3D models are very valuable media for locating the source of deformity and its magnitude ${ }^{21}$. Three-dimensional models can be manipulated in any direction, which gives considerable information to the orthodontist without the need for patient recall or being restricted by the time of clinical assessment ${ }^{27}$. (Fig 2) ${ }^{1}$

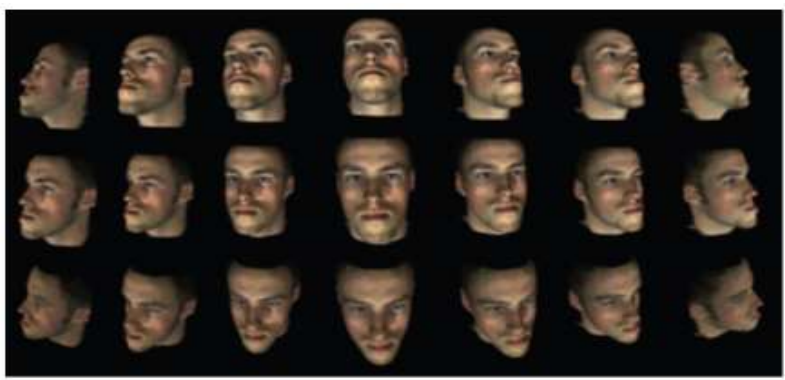

Figure 2: 3D imaging of the face enables the orthodontist to evaluate the face from any direction. Here, a skeletal Class III case is displayed in different views, with gradual rotations around the $y$-axis from $-90^{\circ}$ to $+90^{\circ}$ (middle row). $+30^{\circ}$ and $-30^{\circ}$ rotations around the $x$-axis are shown in the upper and lower rows, respectively

Assessment of outcome can also be performed easily by visual comparison of pre-and post-treatment models placed side by side. (Fig 3) illustrates an example of an Orthognathic patient treated by bi-maxillary surgery, where the face has been scanned using stereophotogrammetry. ${ }^{16,25}$

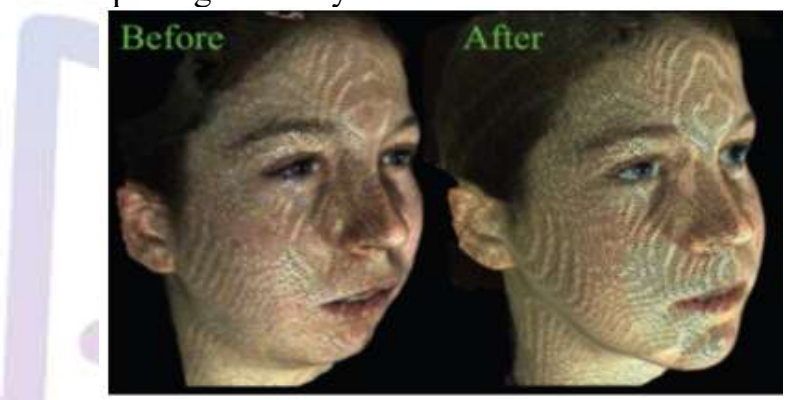

Figure 3. A Skeletal Class II case shown 1 month before and 6 months after Orthognathic surgery ${ }^{1}$

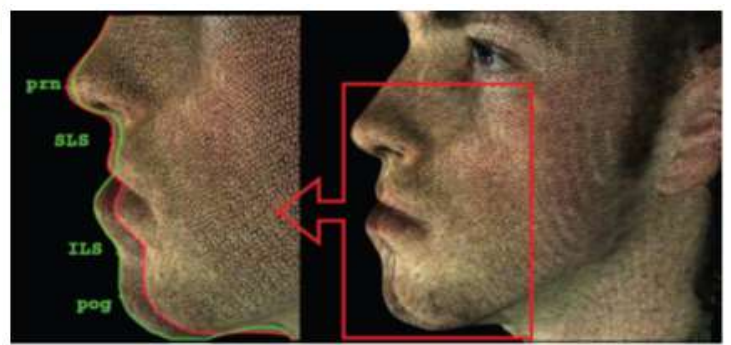

Figure 4. Mathematically superimposed pre- and post-surgical 3D models of a Class III patient ${ }^{1}$.

Communication tool - Instead of discussing treatment objectives and treatment options using X-ray viewers, 2D photographs or composite tracings, life-like 3D models provide a very clear tool for showing areas of deformities, levels of asymmetry and relative relationships between different components of the face, all of which are in an interactive manner on-screen in front of the patient $t^{1,8}$. 
2] Dental imaging:-

A) Intraoral 3D camera

A video camera records the structured light distortions on the dental crowns as it is passed over the dentition over a time period of approximately one minute. The stream of images is fed back to a computer and processed and stitched together to create a complete dental arch. ${ }^{5}$

B) Digital models

When the company receives the impressions and bite registration, the models are poured and scanned through a proprietary process. The maxillary and mandibular digital casts are articulated by using the bite registration that was sent with the impressions. Within 5 days of receiving the impressions, the electronic information is posted on the firm's server as an electronic file. ${ }^{33}$ (fig. $5,6,7,8,9,10,11$ )

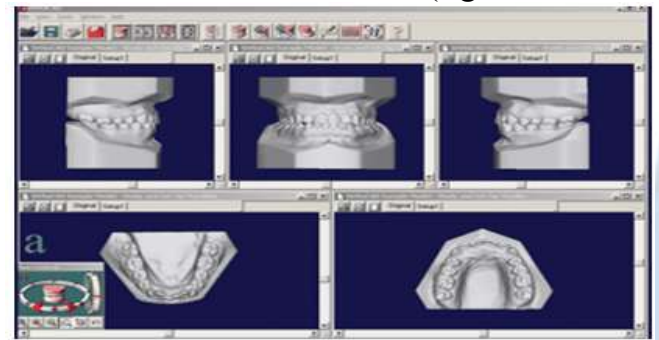

Figure 5: Five simultaneous views of the digital models

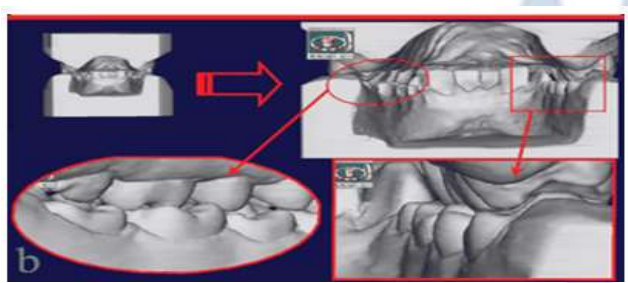

Figure 6: Lingual aspects of the upper and lower teeth can be clearly seen and assessed using OrthoCAD ${ }^{\mathrm{TM}}$ manipulation tools. ${ }^{33}$

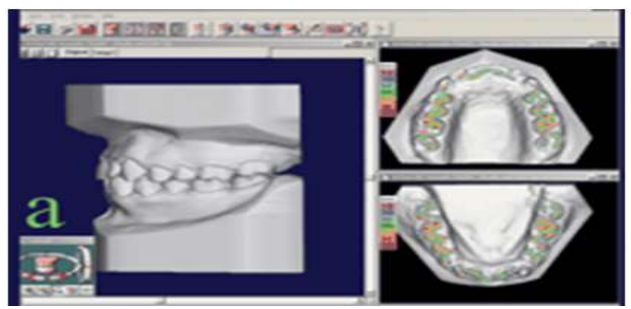

Figure 7: The Occlusogram illustrating the amount of inter-occlusal contacts using color-coded scales

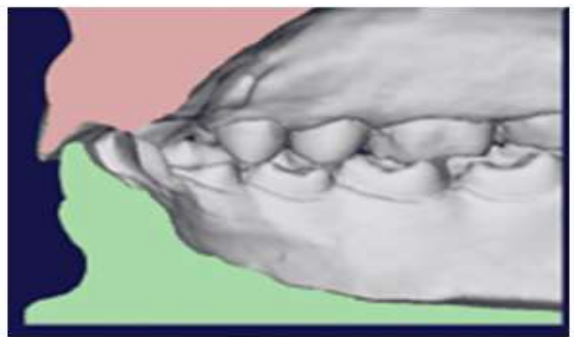

Figure 8: Overbite and overjet can be assessed accurately by splitting the model along the mid-sagittal plane.

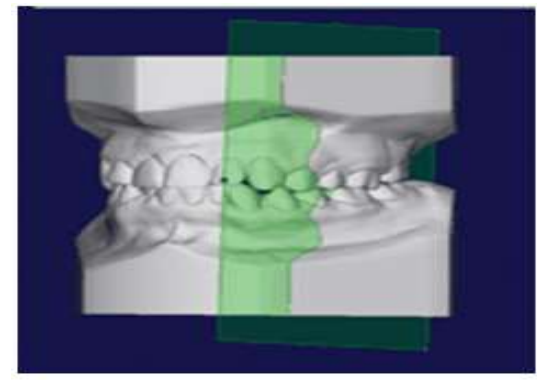

Figure 9: Along with midline analysis, splitting can be performed at any point and in any angle
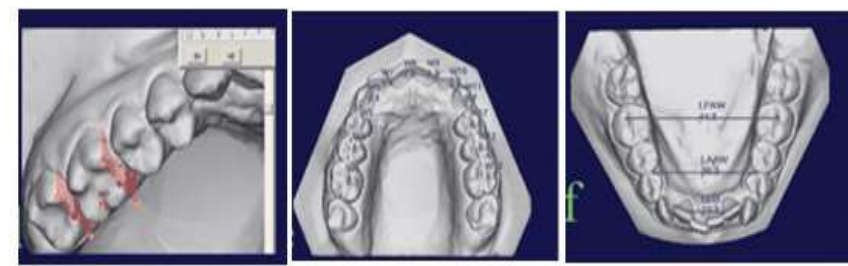

Figure 10: Measuring mesio-distal widths of teeth, space analysis and measurements of arch widths in the lower dentition.

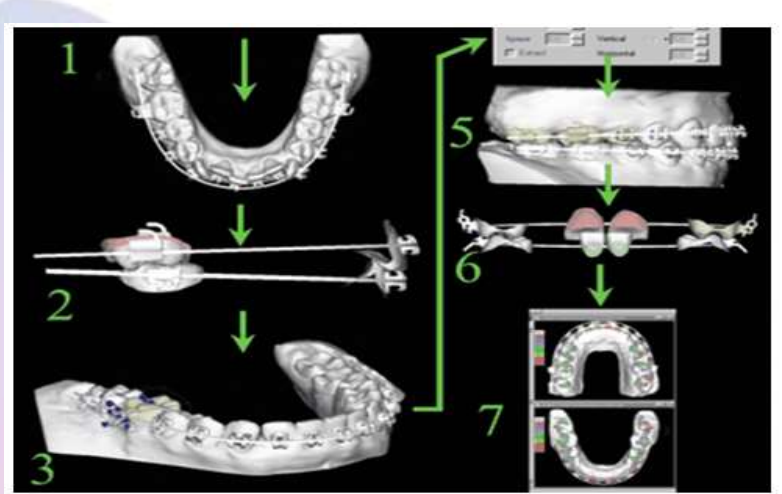

Figure 11: Virtual set-up feature helps us to choose our preferred brackets, bands and wires from the available straight wire systems listed. Secondly, incisors need to be positioned, as well as the molars (if required). In the third and fourth steps, the orthodontist should slide maxillary and mandibular teeth into their proper positions or correct the positions of the brackets themselves to achieve better inter- and intra-arch relationships. Extractions can

be simulated at this stage and the resultant space can be manipulated manually or automatically. In the fifth step, the sagittal inter-arch relationships should be double-checked,

followed by evaluating the transverse relationships in step 6 . Finally, molar position and jaw closure are adjusted to make sure that the correct form of treatment is chosen. ${ }^{14}$

3] Skeletal imaging:-

A) Computed tomography (CT):-

CT uses detectors to catch and measure photons of radiation. A computer then calculates the collected data, measured in one plane at a time, and reconstructs an image called a computed tomogram. 
Clinical applications of computed tomography

1) Identification of landmarks

2) Facial asymmetries

3) Treatment outcome

4) Visualization of individual structures

5) Evaluation of the TMJ

B) Cone beam Computed Tomography (CBCT) ${ }^{10}$ :This technique is a recent development and is found to be more efficient and economical than conventional tomography or CT for oral diagnosis. Cone Beam CT $(\mathrm{CBCT})$ uses a round or rectangular cone shaped X-ray beam centered on a two-dimensional X-ray sensor to scan a $360^{\circ}$ rotation about the patient's head. ${ }^{17}$ Cone Beam Computed Tomography is less expensive than CT, and comparatively free of the labor-intensive costly service. The radiation dose delivered to the patient is only 3 to 20 percent of that of a conventional CT scan. ${ }^{18}$

Uses of CBCT in orthodontics:

1) 3D Cephalometry: In 1994 Jacobson and Gereb developed the 3D cephalometric analysis. 3D cephalometric analysis generated from a cone beam volumetric tomographic scan can now be used to replace 2D cephalometry. CBCT-synthesized cephalograms can be successfully used to perform cephalometric analyses $8,9,31$.

2) Assessment of alveolar bone $e^{7}$ -

Assessment of available bone is necessary prior to arch expansion or labial movement of incisors ${ }^{7}$.

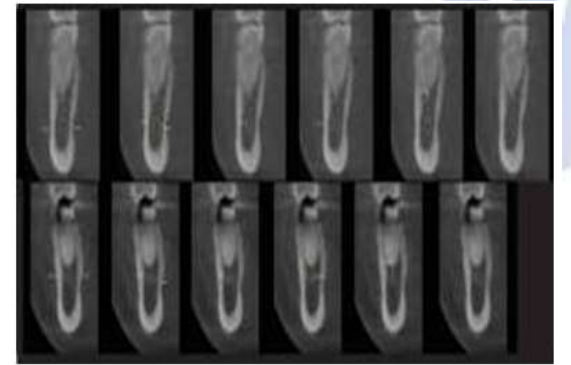

2) Impacted tooth position ${ }^{7}$ :-

Impaction of the teeth is the common orthodontic problem, which requires precise localization for the purpose of surgical exposure and guidance in to the oral cavity. In addition cysts of the jaws, supernumeraries and ectopic teeth can also be visualized using this technique. ${ }^{11}$

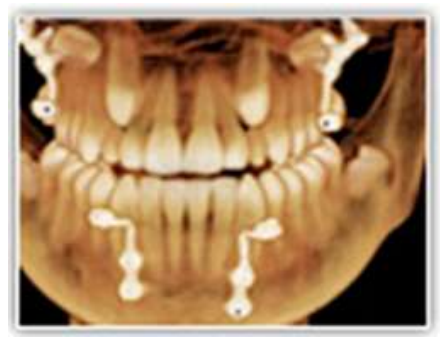

3) Temporomandibular joint assessment ${ }^{7}$

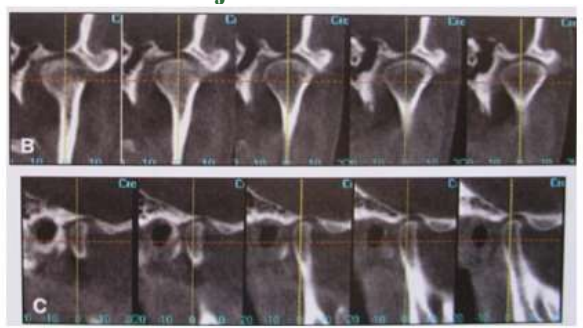

4) Surgical patients and those with syndromes and clefts ${ }^{7}$ :-

Surgical planning for patients with jaw asymmetry, eg: HemifacialMicrosomia can benefit from 3 D imaging? After maxillomandibular advancements, facial soft tissues appear to respond more to the anterior movement of the jaws than they do to increased transverse dimensions ${ }^{35}$. These 3D images are easily rotated and viewed from any angle. This rotating function enables us to precisely analyze asymmetrical facial structures and to clearly visualize structures that cannot be well described with PA cephalometry ${ }^{30}$.

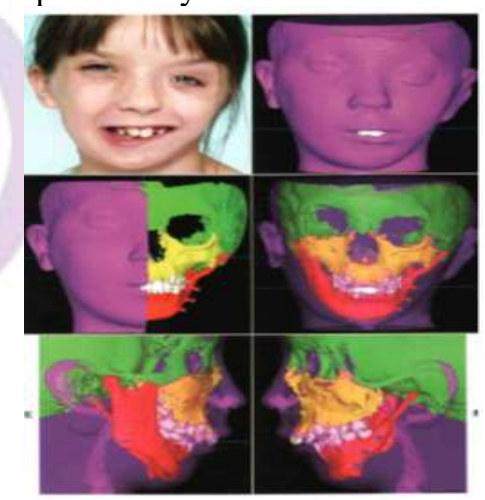

5) Facial analysis :-

The 3D volume can provide any frontal, lateral or user defined view of face and by changing the translucency of the image one can determine the specific relationship of the soft tissues to the skeleton ${ }^{7}{ }^{23}$. Superimposition of cone-beam computed tomography (CBCT) scans by using registration on the whole surface of the cranial base has been validated for nongrowing subjects ${ }^{29,22}$.

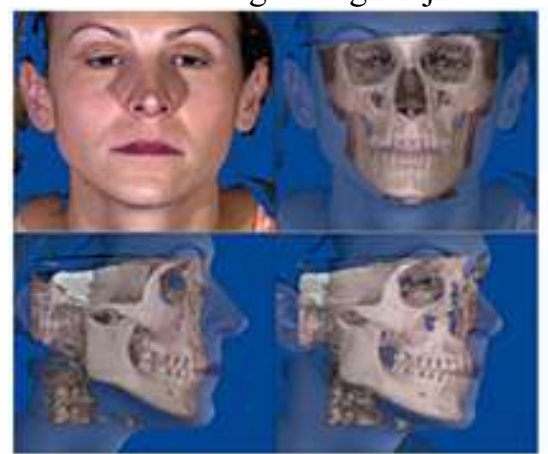


Tongue size and posture ${ }^{7}$ :-

Volume measurements of the tongue could provide a more objective assessment of size, to aid in the diagnosis of open bites and arch width discrepancies ${ }^{7}$.

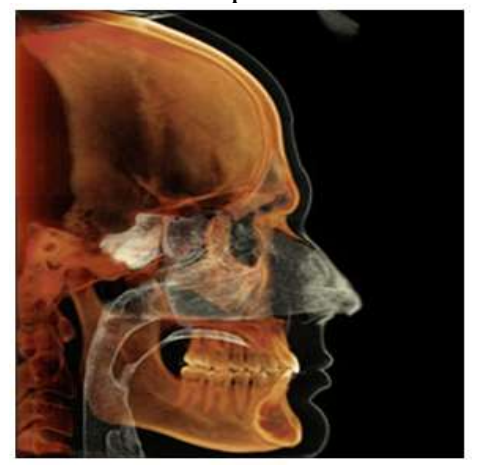

6) Airway assessment ${ }^{7}$ :-

Nasal morphology and turbinates can also be clearly seen in CT scans ${ }^{7}$. Imaging plays a role in the anatomic assessment of the airway and adjacent structures. Obstructive sleep-disordered breathing (OSDB) is not diagnosed with imaging, but imaging can identify patients with airways who are at risk for obstruction and other anatomic characteristics that may contribute to OSDB. The airway extending from the tip of the nose to the superior end of the trachea can be visualized on conventional computed tomography (CT) and cone beam CT (CBCT) scans. Because these scans also include the jaws, teeth, cranial base, spine, and facial soft tissues, there is an opportunity to evaluate the functional and developmental relationships between these structures. The skeletal support for airway is provided by the cranial base (superiorly), spine (posteriorly), nasal septum (anterosuperiorly), jaws, and hyoid bone (anteriorly). The airway valves include the soft palate, tongue, and epiglottis. $^{36}$

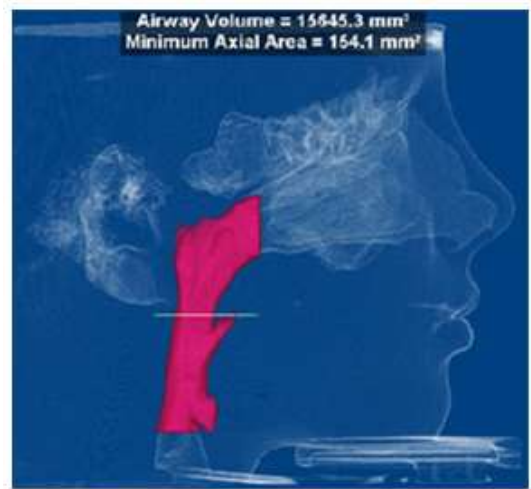

7) Root resorption ${ }^{7}$ :-

3 D CT images can show areas of root resorption on central and lateral incisors adjacent to impacted canine teeth ${ }^{7}$.

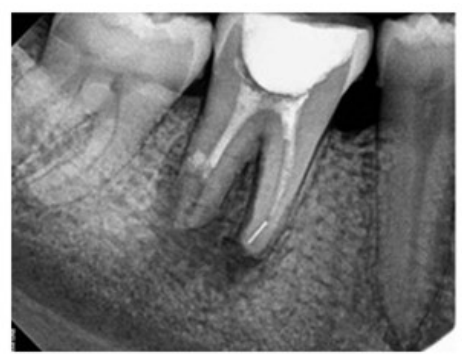

8) Arch length analysis. ${ }^{7}$ :-

Arch length analysis can be accomplished with the data derived from cone beam scans. Arch length is actually three dimensional. The greatest mesiodistal width of individual teeth is measured from the cross sections of the cone beam scans. ${ }^{34}$ The arch form can also be determined from the slices of the cone beam data at the level of the arch. Also, the coronal section through the premolar and molar area allow evaluation of the bucco-lingual position of the teeth in the alveolar bone. Adding these coronal cross section views to the diagnostic workup gives greater insight in to the capacity for expansion and uprighting as well as need to extract teeth or create space with interproximal reduction. ${ }^{7}$
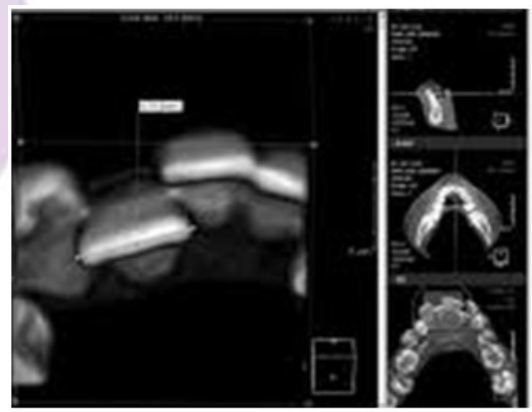

9) Implant site assessment. ${ }^{4}:-$

The greatest impact of CBCT has been on the planning of dental implant placements. CBCT provides crosssectional images of the alveolar bone height, width and angulation and accurately depicts vital structures such as inferior alveolar nerve canal and maxillary sinus.

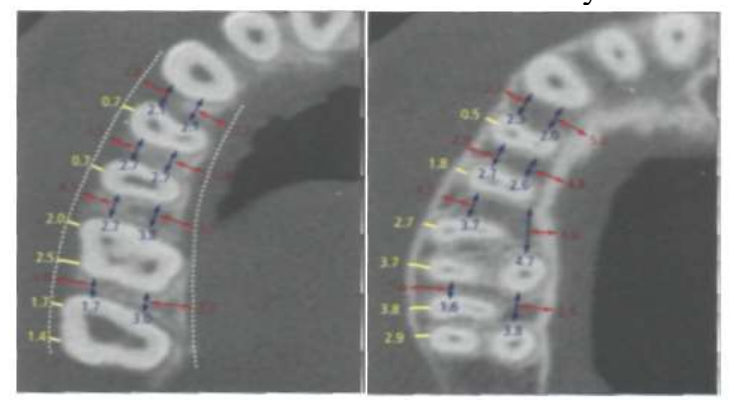




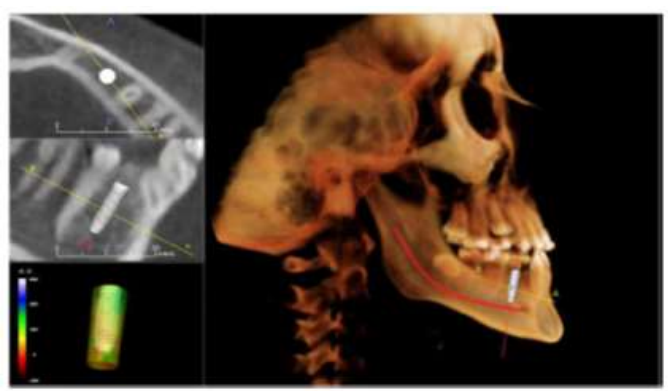

\section{CONCLUSION}

The applications of 3D imaging in orthodontics include pre- and post-orthodontic assessment of dento-skeletal relationships and facial aesthetics, examining orthodontic treatment outcomes with regard to soft and hard tissue prediction, research and medico-legal purposes are also among benefits of using 3D diagnostics in orthodontics. It also allows the clinician to monitor and document the treatment progress and outcome. The 3D imaging has many applications in health care with particular applications in craniofacial growth, orthodontic / orthognathic planning and assessment of treatment outcomes.

\section{REFERRENCES}

1. Hajeer.M.Y, Millett.D.T, Ayoub.F, Siebert.J.P. Applications of 3D imaging in orthodontics: part I. Journal of Orthodontics2004;31:62-70.

2. Hajeer M.Y, Ayoub A.F, Millett D.T, Bock M, Siebert J.P .Three-Dimensional imaging in orthognathic surgery: The clinical application of a new method. Int.J.AdultOrthod.orthognath surgery2002;17:xxx-xxx.

3. Sheldon Baumarind. Integrated Three-Dimensional craniofacial mapping: Background, principles and perspectives. SeminOrthod 2001;7:223-232.

4. Ahmed Ghoneima, EmanAllam, Katherine Kula,Windsor L.J. Three-Dimensional imaging and software advances in orthodontics. www.intechopen.com.

5. James mah, Axel Bumann. Technology to create the ThreeDimensional patient record. Semin Orthod2001;7:251-257.

6. Quintero J.C, Trosien A, Hatcher D, Kapila. Craniofacial imaging in orthodontics; Historical perspective, current status, and future developments. Angle orthodontist 1999; 69: 491-506.

7. AshimaValiathan, Siddhartha Dhar, NikharVerma. 3D CT Imaging in Orthodontics: Adding a new Dimension to Diagnosis and Treatment Planning. Trends.Biomater. Artif.Organs, (2008; Vol 21 (2), pp116-120.

8. LHS Cevidanes, LJ Bailey, GR Tucker Jr. Superimposition of 3D cone-beam CT models of orthognathic surgery patients. Dentomaxillofacial Radiology (2005) 34, 369-375.

9. Demetrios. J. Halazonetics. From 2-dimensional cephalograms to 3-dimensional computed tomography scans. Am J Orthod Dentofac Orthop 2005; 127:627-637.

10. Kau CH, Richmond S, Palomo JM, Hans MG. Threedimensional cone beam computerized tomography in orthodontics. J Orthod. 2005 Dec;32(4):282-93.
11. Waugh RL. Use of Cone Beam Computerized Tomography (CBCT) in orthodontic diagnosis and treatment planning in the presence of a palatally-impacted canine. Orthod Fr. 2014 Dec;85(4):355-61.

12. JM Nervina. Cone beam computed tomography use in orthodontics. Aust Dent J. 2012 Mar;57 Suppl 1:95-102.

13. Chung H. Kau, Jin-le Li, Qingyi Li. Update on Cone Beam Technology and Orthodontic Analysis. Dental Clinics of North America. July 2014; 58 (3): 653-669.

14. C.H. Kau, S. Richmond, J.M. Palomo, et al. Current products and practice three-dimensional cone beam computerized tomography in orthodontics. J Orthod, 32 (4) (2005), pp. 282-293.

15. J.M. Palomo, C.H. Kau, L.B. Palomo, et al. Threedimensional cone beam computerized tomography in dentistry. Dent Today, 25 (11) (2006), p. $130 ; 132-5$.

16. J.K. Mah, J.C. Huang, H. Choo. Practical applications of cone-beam computed tomography in orthodontics. J Am Dent Assoc, 141 (Suppl 3) (2010), pp. 7S-13S.

17. M.B. da Silva, E.F. Sant'Anna. The evolution of cephalometric diagnosis in orthodontics. Dental Press J Orthod, 18 (3) (2013), pp. 63-71.

18. T. Grunheid, J.R. Kolbeck Schieck, B.T. Pliska, et al. Dosimetry of a cone-beam computed tomography machine compared with a digital $\mathrm{x}$-ray machine in orthodontic imaging. Am J Orthod Dentofacial Orthop, 141 (4) (2012), pp. 436-443.

19. B.E. Larson. Cone-beam computed tomography is the imaging technique of choice for comprehensive orthodontic assessment. Am J Orthod Dentofacial Orthop, 141 (4) (2012), p. 402-404, 406 passim.

20. R. Pauwels, J. Beinsberger, B. Collaert, et al. Effective dose range for dental cone beam computed tomography scanners. Eur J Radiol, 81 (2) (2012), pp. 267-271.

21. R.J. Hennessy, S. McLearie, A. Kinsella, et al. Facial surface analysis by $3 \mathrm{D}$ laser scanning and geometric morphometrics in relation to sexual dimorphism in cerebral-craniofacial morphogenesis and cognitive function. J Anat, 207 (3) (2005), pp. 283-295.

22. A.M. Toma, A. Zhurov, R. Playle, et al. Reproducibility of facial soft tissue landmarks on 3D laser-scanned facial images. Orthod Craniofac Res, 12 (1) (2009), pp. 33-42.

23. Z. Fourie, J. Damstra, P.O. Gerrits, et al. Accuracy and reliability of facial soft tissue depth measurements using cone beam computer tomography. Forensic Sci Int, 199 (13) (2010), pp. 9-14.

24. C.H. Kau. Creation of the virtual patient for the study of facial morphology. Facial Plast Surg Clin North Am, 19 (4) (2011), pp. 615-622.viii

25. C.H. Kau, L.M. Hunter, E.J. Hingston. A different look: 3dimensional facial imaging of a child with Binder syndrome. Am J Orthod Dentofacial Orthop, 132 (5) (2007), pp. 704709 .

26. C.H. Kau, S. Richmond. Three-dimensional analysis of facial morphology surface changes in untreated children from 12 to 14 years of age. Am J Orthod Dentofacial Orthop, 134 (6) (2008), pp. 751-760.

27. C.H. Kau, S. Richmond, A. Zhurov, et al. Use of $3-$ dimensional surface acquisition to study facial morphology in 5 populations. Am J Orthod Dentofacial Orthop, 137 (4 Suppl) (2010), pp. S56.e1-S56.e9[discussion: S56-7]. 
28. S.B. Hanis, C.H. Kau, N.M. Souccar, et al. Facial morphology of Finnish children with and without developmental hip dysplasia using 3D facial templates. Orthod Craniofac Res, 13 (4) (2010), pp. 229-237.

29. L.H. Cevidanes, G. Heymann, M.A. Cornelis, et al. Superimposition of 3-dimensional cone-beam computed tomography models of growing patients. Am J Orthod Dentofacial Orthop, 136 (1) (2009), pp. 94-99.

30. H.S. Hwang, C.H. Hwang, K.H. Lee, et al. Maxillofacial 3dimensional image analysis for the diagnosis of facial asymmetry. Am J Orthod Dentofacial Orthop, 130 (6) (2006), pp. 779-785.

31. P.M. Cattaneo, C.B. Bloch, D. Calmar, et al. Comparison between conventional and cone-beam computed tomography-generated cephalograms. Am J Orthod Dentofacial Orthop, 134 (6) (2008), pp. 798-802.

32. K.K. Shi, J.Y. Kim, T.H. Choi, et al. Timely relocation of subapically impacted maxillary canines and replacement of an ankylosed mandibular molar are the keys to eruption disturbances in a prepubertal patient. Am J Orthod Dentofacial Orthop, 145 (2) (2014), pp. 228-237.

33. K.G. Lightheart, J.D. English, C.H. Kau, et al. Surface analysis of study models generated from OrthoCAD and cone-beam computed tomography imaging. Am J Orthod Dentofacial Orthop, 141 (6) (2012), pp. 686-693.

34. A. Gracco, L. Luca, M.C. Bongiorno, et al. Computed tomography evaluation of mandibular incisor bony support in untreated patients. Am J Orthod Dentofacial Orthop, 138 (2) (2010), pp. 179-187.

35. M.S. Ryckman, S. Harrison, D. Oliver, et al. Soft-tissue changes after maxillomandibular advancement surgery assessed with cone-beam computed tomography. Am J Orthod Dentofacial Orthop, 137 (4 Suppl) (2010), pp. S86S93.

36. D.C. Hatcher. Cone beam computed tomography: craniofacial and airway analysis. Dent Clin North Am, 56 (2) (2012), pp. 343-357

Source of Support: None Declared
Conflict of Interest: None Declared 